\title{
Effective complexity: In which sense is it informative?
}

\author{
Esteban Céspedes
}

Valparaíso Complex Systems Institute /

Valparaíso University, Chile

Miguel Fuentes

Valparaíso Complex Systems Institute

Santa Fe Institute/

Draft / version 6.8.19

Forthcoming in the Journal for General Philosophy of Science

\begin{abstract}
This work responds to a criticism of effective complexity made by James McAllister, according to which such a notion is not an appropriate measure for information content. Roughly, effective complexity is focused on the regularities of the data rather than on the whole data, as opposed to algorithmic complexity. McAllister's argument shows that, because the set of relevant regularities for a given object is not unique, one cannot assign unique values of effective complexity to considered expressions and, therefore, that algorithmic complexity better serves as a measure of information than effective complexity. We accept that problem regarding uniqueness as McAllister presents it, but would not deny that if contexts could be defined appropriately, one could in principle find unique values of effective complexity. Considering this, effective complexity is informative not only regarding the entity being investigated but also regarding the context of investigation itself. Furthermore, we argue that effective complexity is an interesting epistemological concept that may be applied to better understand crucial issues related to context dependence such as theory choice and emergence. These applications are not available merely on the basis of algorithmic complexity.
\end{abstract}

Keywords effective complexity, algorithmic complexity, epistemic context, emergence, physical theory 


\section{Effective complexity}

How is it possible to obtain information from very complex things? It seems harder, for example, to know what occurs in cities that are very complex than in small towns. But information hides not only in the complex. We can also ask: How is it possible to obtain information from very simple things? It can also be hard to know what someone means if that person employs a few simple symbols to communicate. Considering this, it is crucial for epistemology in general and for any theory of information to analyze the relations between information and complexity.

According to the notion of algorithmic complexity (also called Kolmogorov Complexity), the complexity of a string of digits or of an expression is equal to the length of the shortest algorithm that may generate it on the basis of a universal computer (Kolmogorov 1965, Chaitin 1969, Li \& Vitányi 1997). The information content of such a string will be its algorithmic complexity. Take again the case of a very complex city. To know better what occurs in it, we may construct a computational model of it. Then, all the information that we can obtain from that model depends on how complex it is, i.e. on how long is the shortest algorithm of a universal computer that can reproduce it. But on which features of the city should we focus to construct the model? Are all their features relevant to define how complex it is? An answer to questions of this kind is found considering a different notion of complexity: effective complexity. According to the account of effective complexity (GellMann \& Lloyd 2003), to determine the complexity of a string of digits we do not focus on the whole string, but on the regularities that we may define on its basis and from a given epistemic context. So, the complexity of an expression is equal to the length of the shortest algorithm that may generate its regularities on a universal computer.

James McAllister (2003) develops a strong argument against effective complexity, trying to show that it is not an appropriate measure for information content. Following the proposal of effective complexity, the information that we may extract from an expression is not equal to its algorithmic complexity. Rather, it is equal to the algorithmic complexity of the regularities that one may focus on, associated with that expression. Now, considering the context-dependence of effective complexity, McAllister concludes that one cannot find unique values of information content for a given piece of data and that, for such a reason, effective complexity is less scientifically suited as a measure than algorithmic complexity. More radically, he also concludes that it is not a useful information measure.

In what follows, we would like to respond to McAllister's criticism, accepting the nonuniqueness of effective complexity, but arguing that we do not have to discard it as a measure of information content. As shown, effective complexity is indeed informative about the expressions to which it is applied, as well as about the scientific context in which it is applied. Additionally, we claim that it is an interesting notion that can be fundamentally helpful to tackle epistemological issues such as theory choice and the analysis of the concept of emergence. In the remaining of this section, we introduce more systematic characterizations of algorithmic and effective complexity. Mcallister's argument is presented in section 2, with a special focus on the supposed problems about the empirical applicability of effective complexity. his second argument (or, rather, the second part of his whole argument) is concerned with the formal aspects of effective complexity. This is the topic of the third section. In the fourth and final section, we develop our arguments about 
the positive aspects of effective complexity, showing in which sense it is informative as well as how it can be applied to problems regarding theory choice and emergence.

Now, let us turn again to the notion of algorithmic complexity. It can be defined in a more formal way as follows:

Algorithmic complexity. The Algorithmic complexity " $\mathrm{K}(x)$ of a finite object $x$ will be defined as the length of the shortest effective binary description of $x . \mathrm{K}(x)$ may be thought of as the length of the shortest computer program that prints $x$ and then halts." (Grünwald \& Vitányi, 2003)

Here is an example that may illustrate this notion: Suppose that someone gives you a sheet of paper in which the pair of letters " $A B$ " is written one hundred times in one line. When you get the paper, you do not know that. This line of letters represents a piece of data, i.e. the object that you have to describe. Even if after a first look it seems that the line only contains $A s$ and $B s$ and in the same order, you may check a few times whether there are other letters in the middle or whether there are two $A s$ or two $B s$ written together. Then you realize that the line is formed by the pair " $A B$ " written one hundred times. After a while someone asks you "What does it say?". You reply "It just says $A B$ a hundred times". By saying that, you have compressed the information contained in the considered object, the line of letters. If you would like to instruct someone to reproduce the same information in another paper, you would not have to dictate each letter one hundred times. You could just say to that person "Write $A B$ one hundred times". Thus, " $A B$ one hundred times" can be considered a short description of the initial piece of information that one can produce in natural language, which, for the sake of the example, may play the role of a universal description method. Considering this, the algorithmic complexity of the information contained in the piece of paper is considerably low, as opposed, for instance, to the information contained in this very sentence.

Thus, it is crucial have in mind that the notion of complexity can provide a definition of information content: The information content of an expression is its algorithmic complexity (see Kolmogorov 1965). Thus, for example, short sentences usually contain less information - and are therefore simpler - than whole books.

Now, it is also important to note that the complexity of an expression can be reduced if it contains patterns. If an expression $a$ contains more patterns than another expression of the same length, $b$, then $b$ should be more complex (and, thus, should contain more information) than $a$. Considering this, the complexity of a string produced by a random process, that is, a string that does not contain any pattern, should be extremely high ${ }^{1}$. As a consequence, we would have to say that randomly produced strings contain a high amount of information. This is problematic, because, intuitively, we neither associate randomness with complexity nor with information (Gell-Mann \& Lloyd 2003). We would expect of expressions containing a high amount of information to say many meaningful things. But a randomly generated string may be completely nonsense. Also, we would expect that, in principle, one could extract a lot of meaningful things from a complex expression, while we may extract nothing meaningful at all from a random string. Facing this problem, Murray Gell-Mann and Seth Lloyd (2003) propose an alternative notion of complexity, called effective complexity.

\footnotetext{
${ }^{1}$ This could be misleading because there are highly compressible strings that contain no patterns (e.g. transcendental numbers like $\pi$ or Euler's number). Strings that represent these objects are extremely compressible, but there is no regularity in such representations. As should become clear later, strings do not contain regularities, in a strict sense. Rather, we can construct ensembles based on those objects and then define regularities based on the relevant similarities that we may observe between the members of those ensembles.
} 
Here is a clear explanation given by Nihat Ay, Markus Müller and Arleta Szkoła:

"The main idea of effective complexity is to split the algorithmic information content of some string into two parts, its random features and its regularities. Then, the effective complexity of [that string] is defined as the algorithmic information content of the regularities alone." (Ay, Muller \& Szkola, 2010)

In other terms, the concept of effective complexity can be characterized as follows:

Effective complexity. Let $I$ be the random component of some expression $a$ and $E$ be its regular component, that is, the component associated with the predictive power of $a$. The effective complexity of $a$ is the algorithmic complexity of $E$.

We should say something more about the idea of regularity considered by Gell-Mann and Lloyd (2003). Suppose that instead of only focusing on $a$, we focus on a set containing entities that are similar to $a$ with regard to certain relevant features or regularities. If we assign probabilities to the members of such a set, we may construct an ensemble. This ensemble is symbolized by $E$ in the definition of effective complexity just given. Thus, the complexity of $a$ is not measured on the basis of its specific details, but on the regular features that give form to the ensemble.

As Gell-Mann and Lloyd argue, a clear understanding of complexity depends on how we draw the distinctions between the features of an entity that are considered as regular and features that are taken as contingent. On this basis, it should be clear that the choice of an ensemble for a given entity is not a procedure that leads to a unique result. Given a particular entity, the set of its relevant features and the set of regularities that one may detect between them vary depending on the epistemic context. As we will see, we do not have to take this as a weakness of the account, but rather as an aspect that allows us to better understand our natural intuitions about complexity, as well as human knowledge in general.

Consider again the example of the line of characters containing the pair " $A B$ " one hundred times. Suppose further that each character is written by hand, such that there are slight graphical differences between them. A key condition that allows you to compress the information contained there and giving the instruction "Write $A B$ one hundred times" is precisely the fact that you recognize the $A s$ and the $B s$ as regularities, even if each instance of the letter $A$ and the letter $B$ is graphically distinct to the other instances of the same letter. We may construct ensembles of possible $A s$ and $B s$ on the basis of the features that all instances of each letter have in common. In this kind of example and perhaps in most cases, algorithmic complexity and effective complexity may produce the same general results. The line constituted by a hundred instances of the pair " $A B$ " has a low complexity according to both measures. However, there are cases in which the two notions differ considerably.

If we are confronted, for example, with a randomly generated expression that does not show any regularity, we may consider it as an extremely simple expression. We cannot construct an ensemble on the basis of such an expression. Therefore, according to the measure of effective complexity, the amount of information assigned to it would be zero. By contrast, following the notion of algorithmic complexity, we should assign a higher degree of complexity to a randomly generated expression, because, in principle, the only thing we can do to reproduce the information contained in it is to write the expression down again. In other words, we cannot compress it. Therefore, the shortest description of such an expression would not be shorter than the expression itself.

A valid question now is whether effective complexity serves as an appropriate measure for information content in general. James McAllister (2003) argues that it is not. His criticism 
is based on two kinds of arguments: an argument based on the application of effective complexity to empirical cases and an argument based on the formal features of that measure. Both arguments depend on the controversial statement that, for any given expression, one cannot assign a unique degree of effective complexity.

Before turning to McAllister's arguments, we would like to note that the effective complexity account is a special case of an account (or cluster of accounts) called two-part code optimization (cf. Li \& Vitányi 1997; Vitányi 2006; Adriaans 2012). Effective complexity is just one of the forms of two-part code optimization that have been framed many times by various authors. A precursor is the work developed by George David Birkhof (1993), who put a great effort in providing a quantitative method to describe aesthetic quality. According to his proposal, aesthetic experience can be divided with regard to formal associations (i.e. measurable properties) and connotative associations (properties that are hard to measure). During the 1970s and 1980s, two-part code optimization got particular attention within information theory and learning theory. Andrey Kolmogorov (1974) defined structure functions which determine stochastic properties of individual data strings (cf. Vereshchagin \& Vitányi 2004). Moshe Koppel (1987) introduced later the notion of sophistication, defined as the size of the part of an object's minimal description to which one can project a structure. According to Koppel, such a notion provides a static characterization of the amount of planning that was necessary to construct the object. As he also shows, sophistification is translatable, under certain conditions, to Charles Bennett's (1982) more dynamic concept of logical depth, which is defined as the running-time of an object's minimal description (cf. Bennett 1995; Antunes et al. 2006). More recent forms of two-part code optimization are given Paul Vitányi's (2006) meaningful information, Wolpert and Macready's self-dissimilarity and Pieter Adriaan's (2012) facticity.

According to the general framework of two-part code optimization, the description of a piece of data can (and maybe must) be expressed in two parts. One part should define the description method itself (the machine, for instance), while the other part should describe the program that reconstructs the data under the interpretation of the given description method. The first part detects regularities within the piece of data. The second leaves out its irregular parts, compressing the initially considered object.Let us take again the example of the paper sheet. As assumed, we can see a line formed by the pair "AB" written one hundred times. The description method we could use in order to instruct someone to reproduce the line may involve ways of detecting letters, ways of considering different drawings as a same letter even when they might have slight graphic differences, ways of expressing those letters phonetically, counting abilities, ways of forming pairs of things, and so on. The description's optimization for the line of characters will not only depend on how a method can detect regularities. This is only the first part. The second part will describe the program, i.e. the functions involved in our ability to say, following our example, "Write this down a hundred times". Note that the program leaves out the irregular parts of the initial piece of data, such as the graphemes' sizes or colors. The shortest program able to reproduce the relevant parts of the line of characters arrives at an optimum description, relative to the given description method $^{2}$. The dependence of the description's suitability on the description method, crucial

${ }^{2}$ As explained, two-part code optimization is a procedure for compressing objects in different ways by selecting different kinds of regularities. One may criticize the generality of this approach arguing that there are ways of compressing data that do not involve finding patterns in it. For example, we may compress the decimal expansion of $\pi$ even if it would pass any randomness test based on density measurements. It is an object that involves a lot of randomness. Now, we do not have to get into this problem in much detail, since our main concern is the question about whether effective complexity offers a good information measure or not. But one brief response to the criticism might go as follows. Consider the set of all known approximations of $\pi$, i.e. the data we have about $\pi$. Every member of that set is similar 
within the two-part code optimization framework, will also be important in our discussion of McAllister's arguments. These are explained in the following sections ${ }^{3}$.

\section{$\underline{2 \text { Its empirical application }}$}

Let us focus on the argument that targets the empirical application of effective complexity, considering an abbreviated version of one of the examples proposed by McAllister:

Atmospheric temperature. A single data set on the earth's atmospheric temperature may be considered on the basis of different time periods. For instance, it may be considered, on one occasion, on the basis of a period of one day and, on another occasion, on the basis of a period of 21,000 years. A pattern may be found on each of both occasions. Depending on different cognitive and practical interests, one may regard one pattern rather than the other as the regular component of the data set. Thus, one cannot assign it a unique degree of effective complexity.

Of course, this case presents a problem if we focus on the notion of effective complexity in general, without specifying how the regular component, $I$, for some piece of information is determined. In order to reply to McAllister's criticism on this point, we may consider the possibility that a particular research agenda may be fixed before the assignment of effective complexity. Such a research agenda involves, among other conditions, a given set of cognitive and practical interests. On this basis, we should consider the example involving atmospheric temperature as follows:

Atmospheric temperature for a particular research agenda. A single data set on the earth's atmospheric temperature may be considered on the basis of different time periods. Suppose that a group of meteorologists restricts the data according to a given research agenda in order to focus on a period of one day. The effective complexity of the initial data set is the effective complexity of the restricted data set.

This way of considering the assignment of effective complexity is clearly compatible with scientific practice and can be applied to other similar cases. One may plausibly expect that two groups of scientists could assign a sufficiently similar effective complexity to a particular data set if their research agendas match appropriately.

Thus, our response to McAllister consists mainly in two points: First, we acknowledge that if the set of cognitive and practical interests is not determined, it is hardly possible to find a unique degree of effective complexity for a given piece of information. This is a crucial property of effective complexity, as Gell-Mann and Lloyd (2003) explicitly establish. Second, if the cognitive and practical interests were determined, we could also (under further assumptions, of course) determine a unique degree of effective complexity or, at least, a sufficiently restricted range of values of effective complexity for a given piece of

to $\pi$ in the fact that, say, it is nearer to 3,14 than to 3,15. This is a regularity. Actually, it is an exceptionless regularity. All members of the set have 1 and 4 as first decimal digits. (This holds even if we include numbers similar to $\pi$ that are not approximations of it). Thus, we are able to compress $\pi$ on the grounds of a regularity shared by all its known approximations.

${ }^{3}$ As may seem clear, we work here on the basis of a very general definition for mainly two reasons: The first is that we want to tackle the issues pointed out by McAllister following, for that purpose, his simple characterization. Second, we think (as McAllister probably thinks as well) that no further technicalities are needed to consider the kinds of arguments discussed here. Anyhow, see the Appendix for a bit more detailed definition. 
information. The set of interests influences the choice of a description method, which, as claimed above, determines the suitability of the resulting descriptions.

We should say a bit more about cognitive and practical interests. We do not mean that effective complexity is necessarily relative to the cognitive and practical interests of individual, cognitive agents. Of course, the set of interests that are relevant for effective complexity may depend on cognitive characteristics of particular agents or research communities. But to define an object's effective complexity it is not essential how interests are physically or psychologically realized, even if they play a crucial role as functions that determine regularities. As Gell-Mann and Lloyd (2003) claim, "[i]n most practical cases, the distinction between regularity and randomness or between regular and random information content depends on some judgment of what is important and what is unimportant, even though the judge need not be human or even alive." The key point is this: A single piece of data may exhibit different regularities and effective complexity can be defined relative to one of those regularities. Each of these regularities can be selected only on the basis of a set of relevance functions. But it does not matter, for the definition of effective complexity, how these functions are physically realized and whether they are cognitive or not, even though our knowledge depends on relevance functions that are cognitive. Some arbitrary functions can be considered as non-cognitive relevance functions. Clearly, this is tricky. For we select non-cognitive relevance functions according to our cognitive and practical interests.

Let us consider this in a somewhat oversimplified metaphysical question: Would there be effective complexity if all cognitive agents were to vanish from the universe? Of course, in some concrete sense, there would not. But metaphysical questions also demand metaphysical answers. We prefer to take a Kantian view here. We cannot say anything certain about the world in itself. But we can grasp an antinomy. On the one hand, if there were absolute, objective laws, then the effective complexity of any entity would depend on the algorithmic complexity of these laws. On the other hand, if the world were just a lawless, chaotic entity, its effective complexity would be zero. Thus, even in this case effective complexity cannot take a unique value.

Let us call "true regularity" a regularity that expresses a law of nature (i.e. a law in the metaphysical sense, not in the scientific sense). Would the conjunction of all true regularities produce a unique effective complexity for any entity, including the world itself? Sure, but the question only has sense under the metaphysical assumption that the world is not really a lawless, single entity. We cannot know, on the basis of empirical reason, whether the world is a lawless entity or not. The best we can do is postulate that it is or that it is not, as a matter of practical reason. We could assume that the world is a single lawless entity, i.e. that there are no true regularities. Again, the effective complexity of the world may vary depending on the metaphysical principles one assumes. It is not unique. Choosing between one of these paths of metaphysical enquiry means choosing according to practical reason, i.e. according to cognitive and practical interests.

These are all hard, metaphysical issues that we might confront while thinking about the notion of effective complexity. However, the arguments that we will put forward are not mainly of this kind. Instead, we would like to put more focus on the epistemological issues related to McAllister's criticism. Let us turn now to the formal aspects of effective complexity.

\section{$\underline{3 \text { Its formal aspects }}$}

The other argument presented by McAllister against effective complexity is focused on the formal aspect of this measure. The main idea of this argument is the general point made in 
the example on atmospheric temperature: There is no unique complexity measure for any given expression. Let $a$ be a string of digits or a piece of information and $R$ be a pattern. Any string of information can be characterized in terms of a pattern and some remaining noise in the following form:

$$
a=R+\text { noise at } n \text { percent. }
$$

The number of possible patterns may be extremely large, because the pattern considered in each occasion depends on the context of the investigation. Following the characterization already given, the effective complexity of $a$ is, roughly put, the algorithmic complexity of $R$. Now, since $R$ is not uniquely determined for any string $a$, the effective complexity of $a$ is not uniquely determined and must be arbitrary. This argument may be reconstructed in a simplified form as follows:

(1) The effective complexity of $a$ is the effective complexity of a regularity that one may extract from $a$.

(2) There is no unique regularity determinable for any given $a$.

(3) Therefore, for any given $a$, the effective complexity of $a$ cannot be uniquely determined.

As McAllister argues, the degree of effective complexity of a given string $a$ is arbitrary. This would mean that effective complexity is not a useful measure of information content. We may respond to this as follows: Effective complexity can be considered to be arbitrary, but just indirectly. It is arbitrary because the determination of regularities is arbitrary, depending on the epistemic context, which may involve a theory used to discriminate regularities from noise (we will discuss this in detail in the next sections). So, if one could determine which is, in fact, the context that determines a particular regularity for a given string and a given subject (or group of subjects), and thus, the theory used, one could also be able to determine a unique value for the effective complexity of that string. Consider the following clarification:

(4) It is possible to determine a unique regularity for any given string and a given context of investigation or theory

(5) Hence, it is possible to determine a unique degree of effective complexity for any given string and a given context of investigation.

Assuming this, the effective complexity of a piece of information can be considered to be non-arbitrary on the basis of some context arbitrarily determined. Indirect arbitrariness of this kind is neither a weakness of the notion of effective complexity nor infrequent in the scientific practice. Take, for instance, any observation process. Observations can be considered as processes of regularity detection, which are determined by human physiology, background knowledge, epistemic interests and technology, among other factors. As is well known, human physiology has evolved on the basis of processes that involve chance. Thus, in a certain sense, it is a statistically arbitrary result. But, of course, this does not mean that observation is not useful. Analogously, the fact that effective complexity is based on arbitrary factors does not mean that it is not a useful measure of information content.

Summarizing the last considerations, we may say, first, that effective complexity is not purely arbitrary, but only indirectly arbitrary and, second, that arbitrariness in this sense must not be taken as a weakness of the notion of effective complexity.

It is true that, if one only focused on the expression under investigation without considering 
the parameters involved in the epistemic context, one would not be able to find a unique value of effective complexity. As well as with regard to its arbitrariness, the non-uniqueness of effective complexity should not be considered as a weakness of the account either. As Gell-Mann and Lloyd (1996) claim, effective complexity represents our intuitions about complexity and corresponds to natural features of human knowledge and learning. Thus, in a very relevant sense, effective complexity can tell us a lot about epistemic contexts and not only regarding the generalities of epistemic contexts. As we will argue in the following section, this notion of complexity may be helpful to determine particular features of epistemic contexts, as well as to contribute to the debate concerning different philosophically important concepts, such as emergence and reducibility.

It must be emphasized, however, that our way of understanding of complexity depends crucially on how we distinguish regularities from the contingent features of an entity. As Gell-Mann and Lloyd show, the foundations of this kind of distinction are mainly epistemic. We think that McAllister's criticism must be tackled having this in mind.

\section{Its effectiveness}

As McAllister claims, his argument shows that effective complexity is not a useful measure of information content. By contrast, one may plausibly think that the same contextdependency that he criticizes is what makes such a measure of complexity especially useful. Let $C$ be a set containing information about epistemic interests, conceptual definitions and factual knowledge. We may call $C$ a context of inquiry. Now let us consider the following general case:

Context choice. Suppose that an epistemic subject $S$ evaluates the effective complexity of $a$ on the basis of $C_{l}$. Among the epistemic interests involved in $C_{l}$, one interest consists in answering some question $q$. Given $C_{l}$, let $R_{l}$ be some regularity attributed to $a$ by $S$. Let $E_{C l}(a)$ be the effective complexity of $a$ on the basis of $R_{l}$. $S$ 's answer to $q$ will depend on that degree of complexity. Suppose further that $S$ tries once again to answer that question, but instead of assuming $C_{1}$, she assumes $C_{2}$. The difference between $C_{1}$ and $C_{2}$ is relevant enough, such that $S$ focuses on $R_{2}$, a different regularity defined on the basis of $a$. Let $E_{C 2}(a)$ be the effective complexity of $a$ according to $C_{2}$. Such a degree of complexity allows $S$ to answer $q$ in a distinct way. Suppose, finally, that, according to some element shared by $C_{1}$ and $C_{2}, S$ judges that $a$ in the light of $R_{2}$ is more appropriate than in the light of $R_{l}$ to answer $q$. Considering this, $S$ may be justified in preferring $C_{2}$ for future questions that may be similar to $q$.

To illustrate this, consider again the example of atmospheric temperature. Suppose that a group of scientists confronts a data set about the earth's atmospheric temperature and wants to investigate (i.e. they ask) how extreme are its fluctuations every 1,000 years during the last 400,000 years. If they focused, in context $C_{1}$, on a time-scale based on daily fluctuations of temperature, they would get more complex regularities than if they focused, in context $\mathrm{C}_{2}$, on fluctuations occurring every 100 years. They can get different answers from different regularities. Which of both sets of regularities is better? Considering that the group is focused on extreme fluctuations of temperature, the set of regularities that they may extract from $C_{l}$ is more relevant and simpler than the other. On this basis, they should focus on contexts like $C_{1}$ (or more similar to it than to $C_{2}$ ) when confronting in the future questions like the mentioned one.

This case should show that effective complexity is actually very useful as a measure of 
information content. Of course, it may not tell us much in absolute terms about an isolated piece of information $a$, but it may tell us much about the context according to which $a$ is being considered at a given point. This feature of effective complexity may be crucial in situations of theory choice, that is, situations in which two different, equally plausible theories or hypotheses are being compared as competitors for the explanation of some set of phenomena. Note that algorithmic complexity cannot be directly applied to this epistemological issue in the same way, because it does not involve the same form of contextdependency.

Note that, since contexts may involve characterizations of theories, cases of theory choice can be treated as special cases of context choice. The case just presented does not involve theory choice necessarily. Now, when theories are considered, context choice might be useful to analyze cases of incommensurability, i.e. cases in which different, incompatible theories are confronted in the search for understanding a common field of phenomena. As should seem clear, however, the example just presented is not about incompatible contexts. The regularities that we might obtain from $C_{l}$ are not incompatible with the regularities that we might obtain from $C_{2}$. Actually, the relevant regularities of $C_{2}$ would be, for the case considered, contained in the set of regularities that are detectable from $C_{1}$. Given a single dataset corresponding to a period of 400,000 years, whatever temperature fluctuations one may observe on the basis of a 100-year scale are also observable on the basis of a daily timescale.

\section{Theory choice}

Let us turn now to the case of incompatible contexts. In some cases, it may not be possible to choose between two incompatible hypotheses $h_{1}$ and $h_{2}$ because both provide suited answers to a given question $q$ (or prediction task). There may be more than one good reason to revise those contexts. Think of simplicity, coherence or parsimony, for instance. At some point it may seem rational to seek a single, coherent theory instead of feeling satisfied with two incompatible ones. Furthermore, there could be a domain of questions, different from the domain of $q$ that could be better investigated on the basis of $h_{1}$ than on the basis of $h_{2}$. In sum, although the applicability of effective complexity may not be directly related to the determination of information content of entities taken in isolation, it may provide, when assigned to a given entity, valuable information about the epistemic context on the basis of which the relevant regularities of that entity were (or can be) extracted.

This is related to the well-known problem of incommensurability (cf. Feyerabend 1962, Kuhn 1962). Roughly, the problem is based on the difficulty of comparing some conflicting theories due to their conceptual incompatibility. For example, classical mechanics is incommensurable with relativistic mechanics. Although both are focused in describing the same type of phenomena (both can offer descriptions from a same piece of data), some terms involved in such descriptions are incompatible. For some questions regarding a certain piece of data-for instance, questions about situations that involve very high velocitiesrelativistic mechanics is more appropriate than classical mechanics. But sometimes, classical mechanics might be more appropriate. A lot will depend on the simplicity of the regularities involved in the answers provided by each theory and this will vary depending on different contextual elements, such as conceptual assumptions, interests and practical procedures. Thus, effective complexity may not give us a unique measure of information about, say, some object moving at a very high velocity, but it may give us (at least) two; one based on classical mechanics and other based on relativistic mechanics. And most importantly, it will give us reasons to choose among different descriptions, depending on the effective 
complexities and the epistemic contexts associated with them. These benefits are not available merely using algorithmic complexity as information measure.

A very interesting point of view connecting the election of a given theory and effective complexity can be found in the work of Ay, Muller \& Szkola (2010). Following their approach, one can see that effective complexity can indeed be used in a way to choose the best theory for a given (physical) phenomenon. In order to understand their conclusion, we have to define first the notion of total information. Roughly, the total information is the sum of the algorithmic complexity and the entropy of the ensemble, which is a measure of ignorance and arbitrariness. Then, on the basis of a theory, the explanation of a given phenomenon should be simple, which implies that its algorithmic complexity should be small. And, obviously, the explanation should not cover all possible outcomes of the ensemble (considering that there is always a certain amount of noise), which means that the entropy associated with the phenomenon should be small. Its effective complexity will then be the infimum of the algorithmic complexity for the set of algorithmic complexities with similar entropy, and that will give us a good approximation of the "best theory" about the phenomenon we want to explain.

This is related to David Lewis' (1973) account of laws based on the notions of simplicity and strength (see also Lewis 1994). According to it, a law of nature is, roughly, a part of the best deductive systems about the set of all true facts. Weakening this idea and instead of considering all true facts, we may just focus on the set of known data. This change may not be enough for defining the notion of a law, but it may be helpful for the evaluation of theories. Lewis' proposal implies a criterion for this kind of evaluation, assuming that theories can be seen as deductive systems. Good theories are the ones that have an appropriate combination of simplicity and strength. We can characterize simplicity as the opposite of algorithmic complexity and strength as the opposite of entropy or ignorance. Let $T$ and $T^{\prime}$ be two competing theories focused on the explanation of the same type of phenomena. If both were equally strong, say, if both had the same predictive power, but $T$ was simpler than $T^{\prime}$, then $T$ should be preferred over $T^{\prime}$. And if both were equally simple, but $T$ had more predictive power, we should consider $T$ as a better theory than $T^{\prime}$. Now, this kind of comparison is not possible without the notion of strength, i.e. without the notion of entropy. This means that this way of determining best theories is only available on the basis of effective complexity and not merely on the basis of algorithmic complexity, which only serves to determine simplicity.

Let us consider again the example of classical and relativistic mechanics. Both are focused on the description and explanation of motion. Let $q$ be a question about the perihelion of Mercury, $d_{N}$ be an answer based on classical mechanics and $d_{R}$ an answer based on relativistic mechanics. Suppose (following the historical development of this rivalry) that $d_{N}$ turns out to be inaccurate, which is interpreted as a weakness of classical mechanics. Adding ad hoc hypotheses may correct the anomalies, but they will just inject more complexity to the theory. Thus, $d_{N}$ is associated with higher complexity and entropy (i.e. lower simplicity and strength), which supports the idea that relativistic mechanics better describes Mercury's perihelion.

Good, simple theories have low algorithmic complexity and their high degree of information content about phenomena is grounded on the fact that they are regularities. In other words, descriptions that are not (based on) regularities cannot be good theories. This is well known and is compatible with how science and knowledge in general develop. If we focused only on algorithmic complexity, descriptions with high information content would be too complex to be considered good theories and simple descriptions would be too poor in information. All this shows that the notion of effective complexity not only grounds criteria for theory choice, but is also a measure of information content that is applicable to a domain 
in which algorithmic complexity alone is not appropriate. This is incoherent with McAllister's claim, according to which effective complexity may not serve as an appropriate measure of information content. Actually, it does and, regarding theories and other kinds of generalizations, it does it better than algorithmic complexity.

Naturally, the improvement of descriptions, theories or models concerning a given piece of information might be achieved by reducing their complexity. Theodor Leiber (1999) considers, on the basis of a notion of effective complexity, how theoretical scientists often eliminate aspects from computationally intractable models, sometimes changing the interpretation of their variables, their relations and empirical validations. The main aim of these procedures, called by Leiber complexity reductions, is to make tractable the problems that were not computable within the framework of the older models. The notion of effective complexity used by Leiber is understood in terms of the (computational) costs that are needed for the resolution of some task (for instance, prediction or reproduction of information). The complexity of a task can be defined as the minimal cost of all theoretical approximations, considering also a factor of error, which cannot be informationally greater than the information contained in the task (see Leiber 1999, p. 90). A crucial point in Leiber's proposal is the idea that complexity reductions are always carried out on the basis of explicitly constructed reduction vehicles, which can be viewed as intermediating, theoretical premises, i.e. as epistemic contexts, in a certain way. Thus, the fact that complexity reductions generate soluble models supports the explanatory and scientifically relevance of effective complexity we want to emphasize.

Here is a crucial question: How can effective complexity be used to make choices between incompatible theories? One should expect that incompatible theories succeed in identifying different regularities from the data, which are associated with different effective complexities. In this sense, one also expects that these theories avoid high levels of randomness associated with the given piece of data. Again, theory choice is about tradingoff simplicity and strength. And these are two fundamental notions that constitute the definition of effective complexity. We should have in mind that this identification of regularities does not have to be considered as a "discovery" of the world's regularities, but it can be seen as an epistemic interpretation of the data. We do not have to assume anything about a fixed nature ordered by fixed regularities, i.e. laws in a strict, ontological sense. Only the object under study (a data piece, for example) may be assumed as fixed at a given point during research. But it is also assumed that the data obtained from a certain field of phenomena is always growing and changing. This is compatible with a Kantian perspective according to which reality in itself, considered as the source of the data, is unknowable. Thus, whether such a reality involves fixed regularities or not is beyond our knowledge. The best we can do is postulate our best regularities as if they were real and construct our ontologies accordingly. But again, our argument is more focused on epistemological aspects of effective complexity than on metaphysical ones.

\section{Emergence}

A conceptual field in which effective complexity may also be fruitful is the field concerning emergent properties. According to a general characterization, an emergent property can be understood as follows (see el-Hani and Pereira 2000, p. 133). Given a system constituted of material particles, an emergent property is a property of that system that arises when the interactions between its particles reach a certain level of complexity. Some examples of emergent properties are climate phenomena, economic crises and mental states. We may call emergence the process by which an emergent property arises from the constituent elements 
of a system. Considering that the notion of complexity is crucial in order to understand emergence, it seems natural to provide a definition of an emergent property in terms of a complexity measure. Consider the following definition based on effective complexity ${ }^{4}$ (cf. Fuentes 2014):

Emergent property. A property $P$ of some entity $x$ is an emergent property just in case, on the basis of $P$, the effective complexity of $x$ increases (or decreases) abruptly.

One crucial idea of this way of characterizing an emergent property is the fact that the abrupt increment of effective complexity must be a consequence of some change within what we may call the epistemic context according to which the system is being investigated (as noted in the discussion above related to the election of a good theory). As mentioned, such a context may involve or be about a set of theories, definitions and relevant facts. Another important feature of this notion of emergence is related to the fact that emergent properties may arise as a result of small contextual changes.

It could be interesting now to focus on two main features that are usually associated with emergence and point out briefly how they are expressed by the notion of emergence just characterized. One of these features is novelty. Emergent properties are new properties of a system, properties that were neither observable during the system's initial stages nor describable in terms of its components. Since the effective complexity of an entity depends fundamentally on the regularities that one may extract from it, a change of the effective complexity measure of an entity implies a change of focus regarding the regularities considered. Now, every regularity can be described in terms of properties or relations. Thus, a change with regard to a regularity implies a change of the properties that one is considering. Such a change may be the introduction of a new property or a definition change regarding a previously considered property.

Note that we may characterize the notion of an emergent property in a similar way just on the basis of algorithmic complexity. However, it would not be as rich as the notion defined in terms of effective complexity ${ }^{5}$. Cases of emergence involving, for instance, a change of epistemic interests could not be studied as one could do it on the basis of effective emergence.

The second main feature on which we want to focus is irreducibility. Let $E_{C l}(a)$ be the effective complexity of a system $a$, according to a given context $C_{1}$ concerned with microscopic descriptions and let $E_{C 2}(a)$ be the effective complexity of $a$, according to a given context $C_{2}$, which is concerned with macroscopic descriptions. Also, let $R_{1}$ and $R_{2}$ be the regularities that generate $E_{C 1}(a)$ and $E_{C 2}(a)$ respectively. In some cases, a change from $C_{1}$ to $C_{2}$ may imply that $E_{C 2}(a)$ exceeds $E_{C 1}(a)$ considerably. This occurs when an emergent property is observed. Let $P$ be the emergent property involved in $R_{2}$ that produces the abrupt increment of effective complexity. Then, for any description $q$ involving $P$ and for any description $r$ of $C_{l}, q$ cannot be reduced to $r$. This notion of emergence depends on how we represent the world and, in this sense, may be called epistemological or representational emergence, following Olivier Sartenaer's (2016) terminology. Also, emergent properties characterized on the basis of effective complexity are compatible with what he calls

\footnotetext{
${ }^{4}$ Dramatic shifts in complexity have also been studied extensively in the context of algorithmic complexity and two-part code optimization on the basis of Kolmogorov's structure functions.

${ }^{5}$ Consider the extreme case in which a process suddenly starts behaving in a completely random way. Its algorithmic complexity would increase abruptly. But should we see this as a sign of an emergent property? It seems plausible to think that randomness, as such, could be considered as emergent here. There would also be a decrease of effective complexity, given the loss of regularity, and emergence would be present in this sense too. However, on the basis of effective complexity, we can also say something about the relevant emergent properties that are associated with the increasing randomness.
} 
explanatory emergence, according to which the high-level behavior of a system cannot be explained adequately only on the basis of the behavior of its parts. The main point of this feature lies in the relevance of epistemic contexts for the description of emergent phenomena.

Note now that if we decided to define emergence only on the basis of algorithmic complexity, the irreducibility criterion would be hard to satisfy ${ }^{6}$. Since, in principle, one can find a unique value of algorithmic complexity for any entity, the anomalous increment of an entity's complexity could be seen as independent from epistemic context (as we characterize it) and determined fundamentally on the basis of how the expression describing it is computed at different times. In other words, one would be able to explain the high-level behavior of a system mainly on the basis of the behavior of its parts (i.e. the parts of the expression that describes it).

Here is a brief example of emergence understood in terms of effective complexity. We may study a living organism looking at its molecular components, according to a microscopic perspective. Now, if we change from such a perspective to another context focused on macroscopic features, the complexity of the system may impress us and the concepts associated with a living behavior may change crucially. The property related to the new concept of being a living organism can now be considered as an emergent property. Descriptions involving that property, which are still based on the macroscopic observable behavior of some systems, cannot be reduced to any set of descriptions based on the molecular composition of those systems. Of course, we may tell a complete microscopic story about the molecular dynamics underlying living behavior, but this does not mean that living behavior, as an emergent property, can be strictly reduced to that story.

\section{$\underline{5 \text { Conclusions }}$}

In this work we have argued in favor of the (useful) properties of the so-called effective complexity measure. An important, crucial, point is that effective complexity depends on the distinction between features of an entity that are considered as regular and features that are taken as noise or accidents. This distinction is related to some particular, epistemically contextualized theory used in order to understand the phenomena (the string of data). We have argued that this characteristic is one of the strengths of effective complexity. As shown, it can be of great help in theory choice, for example. As well, if the cognitive and practical interests were determined for a given set of data, one could also determine a unique degree of effective complexity in a relevant and precise way for that research context.

Another valuable aspect associated with effective complexity, we argued, is that it can be used in different philosophical fields. For instance, it can ground a notion of emergence, more specifically epistemic emergence. Using the definitions discussed in this work, one can see how a radical change in effective complexity can be considered as a sign of an emergent phenomenon. Indeed, if a property or phenomenon can be understood using a theory that gives us a small value of effective complexity for a given set of parameters (the technically called "control parameters") and, for another set, it gives us a much higher value, we can say that the phenomenon is emergent (using that theory). Thus, effective complexity can be a qualitative and quantitative measure to obtain information about epistemic emergent properties.

\footnotetext{
${ }^{6}$ Although the irreducibility criterion may not be satisfied for one-part code optimization, based on algorithmic complexity, it could be satisfied using Kolmogorov's structure function or other forms of two-part code optimization (cf. Kolmogorov 1974; Li \& Vitányi 1997; Vereshchagin \& Vitányi 2004).
} 
Although McAllister's arguments on the notion of effective complexity are clear, we wanted to address complementary features that enrich the discussion on it as what it is, namely an informative measure.

\section{$\underline{\text { References }}$}

Adriaans, P. (2012). Facticity as the amount of self-descriptive information in a data set. arXiv preprint arXiv:1203.2245.

Ay, N., Muller, M., \& Szkola, A. (2010). Effective complexity and its relation to logical depth. IEEE transactions on information theory, 56(9), 4593-4607.

Bennett, C. H. (1982). On the logical "depth" of sequences and their reducibilities to random sequences. IBM report.

Bennett, C. H. (1995). Logical depth and physical complexity. In Herken, R. The Universal Turing Machine A Half-Century Survey. Springer.

Birkhoff, G. D. (1933). Aesthetic measure. Cambridge.

Chaitin, G. J. (1969). "On the Simplicity and Speed of Programs for Computing Infinite Sets of Natural Numbers". Journal of the ACM 16 (3): 407-422.

El-Hani, C. \& Pereira, A. (2000). Higher-level descriptions: why should we preserve them. In Andersen, Emmeche, Finnemann \& Christiansen (eds.). Downward Causation. University of Aarhus Press.

Feyerabend, P. (1962). Explanation, reduction and empiricism. In Feigl, H. \& Maxwell, G. (eds.). Scientific explanation, space and time. Minnesota Studies in the Philosophy of Science. University of Minnesota Press.

Fuentes, M. 2014. Complexity and the Emergence of Physical Properties. Entropy 16 (8): 4489-4496.

Gell-Mann, M. \& Lloyd, S. (1996). Information Measures, Effective Complexity, and Total Information. Complexity.

Gell-Mann, M. \& Lloyd, S. (2003). Effective Complexity. Santa Fe Institute Working Papers. 387-398.

Grünwald, P. D., \& Vitányi, P. M. (2003). Kolmogorov complexity and information theory. With an interpretation in terms of questions and answers. Journal of Logic, Language and Information, 12(4), 497-529.

Joosten, J., Soler-Toscano, F. and Zenil, H. (2011). International Journal of Unconventional Computing 7( 5): 353-387. 
Kolmogorov, A. N. (1965). "Three Approaches to the Quantitative Definition of Information”. Problemy Peredachi Informatsii 1(1): 3-11.

Kolmogorov, A. N. (1974). Complexity of algorithms and objective definition of randomness. Uspekhi Mat. Nauk, 29(4), 155.

Koppel, M. (1987). Complexity, depth, and sophistication. Complex Systems, 1(6), 10871091.

Kuhn, T. (1962). The Structure of Scientific Revolutions. The University of Chicago Press.

Leiber, T. (1999). Deterministic chaos and computational complexity: The case of methodological complexity reductions. Journal for General Philosophy of Science 30 (1):87-101.

Lewis, D. (1973). Counterfactuals. Blackwell.

Lewis, D. (1994). Humean supervenience debugged. Mind, 103(412), 473-490.

Li, M. \& Vitányi, P.M.B. (1997). An Introduction to Kolmogorov Complexity and Its Applications. Springer. Second Edition.

McAllister, J. (2003). Effective complexity as a measure of information content. Philosophy of Science 70 (2):302-307.

Sartenaer, Olivier (2016). Sixteen Years Later: Making Sense of Emergence (Again). Journal for General Philosophy of Science 47 (1):79-103.

Vereshchagin, N. K., \& Vitányi, P. M. (2004). Kolmogorov's structure functions and model selection. IEEE Transactions on Information Theory, 50(12), 3265-3290.

Vitányi, P. M. (2006). Meaningful information. IEEE Transactions on Information Theory, 52(10), 4617-4626.

Wolpert, D. H., \& Macready, W. (2007). Using self-dissimilarity to quantify complexity. Complexity 12(3), 77-85. 


\section{$\underline{\text { Appendix }}$}

We would like to show here, following Gellman and Lloyd (1996, 2003), a more specific way to characterize effective complexity, based on steps, as follows:

Effective complexity. In order to obtain the effective complexity of a string $a$ we can, according to a theory or an epistemic context $C$,

first, construct an ensemble $E$ on the basis of $a$, i.e. a set of entities that are similar to $a$, according to $C$,

second, determine the random part of $E$, according to $C$, as well as its regular part, and, finally, assign, according to $C$, a value of algorithmic complexity to the regular part of $E$.

The value obtained with this procedure is the effective complexity of $a$, according to a particular epistemic context. Each member of an ensemble is assigned a probability, such that the ensemble's probability distribution expresses the regularities associated with $a$. On the basis of that distribution, $a$ 's effective complexity, according to $C$, is the algorithmic complexity of $E$ 's regular part. Epistemic contexts may involve different constraints regarding the description of $a$, considerations about other theories that may describe $a$ and information about members of $E$ other than $a$, given the considered regularities expressed by the ensemble. 